\title{
Declaring independence: why we should be cautious
}

A review article identified 246 different risk factors for coronary heart disease. ${ }^{1}$ Many of these 246 were risk factors asserted to be "independent", that is, their association with coronary heart disease was not due to relationships with other, confounding, factors. Establishing the independence of effects is central in analytical epidemiological studies and is seen as an important step in assigning causality to a relationship. But even given the obsession epidemiologists have for multifactorial theories of disease causation ${ }^{2}$ it seems unlikely that there actually are 246 separate causes of coronary heart disease. What might be going on here?

Consider two examples. We are told that poor dental health ${ }^{3}$ and coffee consumption ${ }^{4}$ are both independent risk factors for coronary heart disease. In these cases confounding clearly occurs. Smoking and socioeconomic position are strongly related both to dental health and to coronary heart disease risk; similarly smoking, type A behaviour, and obesity are related to coffee consumption. Thus drinking 10 or more cups of coffee per day was associated with a relative risk of myocardial infarction of 4.9. After adjusting for confounding variables this fell to 2.9 , which was still significantly greater than 1 . In these papers, as in countless more, the authors declare that a factor is independent because a significant association remains after "adjusting" or "controlling" for the confounding factors in multiple logistic regression models. We discuss here the assumptions made in concluding that a risk factor exerts an independent effect on the basis of such statistical results. In particular we are concerned with error and misclassification which occurs when risk factor levels are measured.

Consider the situation with a risk factor of interest and a single confounding factor. Imagine that the relative risk for the association between our risk factor and some disease is significantly different from unity, after adjustment for the confounding factor. When concluding that the effect of the risk factor is independent of the confounding factor it is assumed that the fitted model is correct. With a typical logistic model this would mean first, that the relative odds increased exponentially with increasing levels of any continuous risk factor; and second, that the relative odds associated with the risk factor of interest were the same at every level of the confounding factor so that no interaction existed.

A third important assumption made when concluding that the effect of the risk factor is independent of the confounding factor is perhaps less widely appreciated than these last two. Logistic models assume that the measured values of the risk factor and the confounder correctly characterise the true, or usual, exposure level. In the case of categorical risk factors this means that no subjects are misclassified. Clearly most epidemiologists are aware that this assumption is at least to some extent incorrect, but the problem is often ignored.

So what effect can error in measuring exposure level (for continuous risk factors) or misclassification (for categorical risk factors) have on multivariate relative risk or odds ratio estimation? This will depend on whether such error is systematic (eg, a tendency for everyone to be recorded with a lower weight than their true weight, perhaps due to faulty scales) or random (eg, a subject's weight level is no more likely to be recorded too high than too low). If the error in measurement of a risk factor is random then the estimated relative risk relating the factor to the risk of disease will be biased towards unity. For an exposure which increases the risk of disease, and is thus associated with a relative risk greater than 1, random error in the measurement of the exposure will lead to underestimation of the relative risk.

Statisticians have long recognised that random measurement error leads to underestimation of the size of regression coefficients, but the problem has been neglected in the epidemiological literature. Recently however, the way in which the use of single measurements of blood pressure leads to underestimation of the strength of the association between blood pressure and incident coronary heart disease has been discussed. ${ }^{5}$ The authors describe a method for estimating the degree to which random measurement error leads to weakening of the association between blood pressure and coronary heart disease. They conclude that the regression coefficient linking diastolic blood pressure to coronary heart disease risk would be about $60 \%$ greater if there was no error in the measurement of blood pressure. They refer to attenuation of regression coefficients due to random measurement error as "regression dilution bias". Such attenuation could equally be due to errors in measurement, or to intraindividual variation in the level of a factor, so that a single measurement would serve as a poor proxy for exposure over a lifetime.

Clearly such "regression dilution bias" is important when considering univariate, unadjusted relative risks. However it can be particularly important when considering the effect of a risk factor on risk of disease after adjustment for a confounding factor. If the risk factor and the confounding factor are correlated to any substantial degree then the extent of error in measurement in either the confounding or the risk factor can significantly affect the adjusted relative risk estimate. ${ }^{6}$ In the case of coffee and myocardial infarction, mentioned above, the relative risk associated with drinking 10 or more cups of coffee per day fell from 4.9 to 2.9 after adjustment for confounding variables in a multiple logistic regression model. ${ }^{4}$ The single measurements taken of the confounders are a poor proxy for the lifetime experience of the subjects, and will also suffer from some degree of simple measurement error. Thus their effect on risk will have been underestimated. If the confounding factors could have been better characterised then the relative risk for coffee drinking might have fallen to 1 after adjustment. In other words, it can appear that a risk factor is related to risk of disease after adjustment for confounding factors, but this residual relationship only exists because of underadjustment for these confounding factors.

The epidemiology of cervical cancer provides another example of how poor measurement of confounding factors may leave residual confounding. Many studies report that cigarette smoking is associated with risk of cervical cancer. An important confounding factor is exposure to some sexually transmitted agent, probably viral, which is related to the development of cervical cancer. The risk of exposure to the sexually transmitted agent has been indexed in these studies by reported age at first intercourse and/or reported lifetime number of sexual partners. Clearly these are very poor proxy measures of actual contact with the sexually transmissible agent, and there is thus a huge amount of error in classifying subjects' exposure to this major confounding factor.

Cigarette smoking is strongly correlated with reported sexual behaviour. In most studies the positive association 
between cigarette smoking and risk of cervical cancer is reduced after adjustment for lifetime number of sexual partners and/or age at first intercourse, but remains positive and statistically significant. Thus in one study the crude odds ratio associated with being a current smoker was $10 \cdot 1(95 \%$ confidence intervals $7 \cdot 0-14 \cdot 7$ ), which was reduced to 3.4 $(2 \cdot 1-5 \cdot 6)$ after adjustment for the confounders in a multiple logistic regression model. ${ }^{7}$ It has been reported that since there are 26 studies which find a significant association between smoking and cervical cancer, many of which have included adjustment for confounders, the evidence for a causal effect is now overwhelming. ${ }^{8}$ Unfortunately, if a bias exists, replication of studies cannot eliminate it. This is not to say that the observed "independent" association between smoking and cervical cancer is completely accounted for by underadjustment for exposure to a sexually transmitted agent, only that the methodologies used cannot tell us one way or the other. Indeed such are the problems of confounding in the countries-generally the USA and in Europe-where most studies have been undertaken, that it would not increase our knowledge if 26 more studies were amassed. It would be more useful to carry out just one study in a location where the confounding does not occur, because of different cultural associations of cigarette smoking. Interestingly a case-control study already carried out in Lesotho ${ }^{9}$ is probably the nearest we have come to this ideal. That investigation found no association between cervical cancer and smoking.

A further example comes from the study of coronary heart disease. It is now widely believed that serum high density lipoprotein cholesterol (HDL-C), has an important inverse relationship with risk of coronary heart disease. A potentially important confounding factor for this relationship is serum triglyceride level. Triglyceride levels have a strong, positive association with risk of coronary heart disease. Furthermore there is a high inverse correlation between HDL-C and triglyceride. Evidence from prospective studies suggests that the association between HDL-C and risk of coronary heart disease is attenuated only slightly after adjustment for serum triglyceride in a multiple logistic regression model. In contrast the association between serum triglyceride and risk of coronary disease almost disappears after adjustment for HDL-C.

Serum triglyceride concentrations vary considerably during the day, from day to day, and from month to month. This means that if only one measure is taken, it poorly characterises a subject's true, or usual level. In turn, this means that the association between serum triglyceride and risk of coronary heart disease tends to be substantially underestimated. On the other hand, HDL-C is less variable, with a single measure better characterising a subject's usual level. The observed association between HDL-C and risk of coronary disease is therefore less of an underestimate. Due to this imbalance in the degree to which single measures of HDL-C and triglyceride characterise the usual exposure status, the association between HDL-C and the risk of coronary heart disease has been underadjusted for the confounding factor, triglyceride. Similarly the fact that the relationship between triglycerides and coronary disease disappears after adjustment for HDL-C may reflect the strong correlation between triglycerides and HDL-C, and the greater precision of measurement of HDL-C; thus, paradoxically, the situation arises in which measurement of HDL-C is better characterised by a subject's usual triglyceride level than by a one off direct measurement of triglyceride. In this situation the disappearance of the association between triglycerides and coronary heart disease may be due to differential error in measurement of the two factors, and not due to triglycerides having no influence on coronary disease risk.

Again it must be concluded that prospective studies in which these lipid factors have been measured only once cannot reliably disentangle the separate independent effects of the various lipids. HDL-C may well be an important risk factor in coronary heart disease, and triglycerides may be unimportant, but it would be dangerous to conclude this on the basis of the observational studies alone.

There is, therefore, danger in concluding that the association between one factor and the risk of disease is independent of another, simply on the basis of fitting a multiple logistic regression model. Findings from outside epidemiology reveal the fallacy of such associations. The use of amyl nitrite "poppers", an effective method of increasing sexual arousal which was popular among gay men in the USA, ${ }^{10}$ was initially considered to be a possible cause of AIDS. A large relative risk remained associated with the use of poppers after adjustment in a multiple logistic model for the confounding effects of number of sexual partners and range of sexual practices. ${ }^{11}$ The use of poppers was almost certainly related to the risk of exposure to HIV. However risk of exposure to HIV would be poorly indexed by reports of number of sexual partners and types of sexual practices. Thus the identification of HIV led to the poppers theory being dropped, even though some epidemiological studies continued to find it to be an independent risk factor. ${ }^{1213}$ As one commentator has pointed out, wearing Levi $501 \mathrm{~s}$ or owning the "Judy Garland Live at the Carnegie Hall" recording would probably also be found to be "risk factors" for AIDS. ${ }^{14}$ They could, indeed, be "independent" risk factors.

There are obvious parallels between the example of poppers and AIDS and that of smoking and cervical cancer. If HIV had not been identified there could well now be 26 studies showing poppers to be an "independent" risk factor for AIDS, and reviews could be published expressing surprise that the relationship had not been accepted by all authors as causal.

Epidemiologists must accept that there are situations where a major confounding factor is subject to so much measurement error that the methodology is inadequate to address the question at hand. Otherwise we may be stuck with the intensive investigation of artefacts, which many of the current "independent effects" may well be.

George Davey Smith

Department of Epidemiology and Population Science London School of Hygiene and Tropical Medicine

Andrew Phillips

Department of Public Health and Primary Care Royal Free Hospital School of Medicine

London

1 Hopkins PN, Williams RR. A survey of 246 suggested coronary risk factors. Atherosclerosis 1981; 40: 1-52.

2 McCormick J. The multifactorial aetiology of coronary heart disease: a dangerous delusion. Perspect Med Biol 1988; 32: 103-8.

3 Mattila KJ, Nieminen MS, Valtonen VV, et al. Association between dental health and myocardial infarction. $\mathrm{Br} \mathrm{Med}$ f 1989; 298: 779-82.

4 Rosenburg L, Palmer JR, Kelly JP, Kaufman DW, Shapiro S. Coffee drinking and nonfatal myocardial infarction in men under 55 years of age. Am $₹$ Epidemiol 1988; 128: 570-8.

5 MacMahon S, Peto R, Cutler J, et al. Blood pressure, stroke and coronary heart disease. Part 1, prolonged differences in blood pressure: prospective heart disease. Part 1, prolonged differences in blood pressure: prospective 1990; 335: 765-74.

6 Armstrong BG, Whittemore AS, Howe GR. Analysis of case-control data with covariate measurement error: application to diet and colon cancer. Stat Med 1989; 8: 1151-63.

7 Slattery ML, Robison LM, Schuman KL, et al. Cigarette smoking and exposure to passive smoke are risk factors for cervical cancer. $\mathcal{F} A M A 1989$; 261: $1593-8$.

8 Winkelstein W. Smoking and cervical cancer-current status: a review. $\operatorname{Am} \mathcal{F}$ Epidemiol 1990; 131: 945-57.

9 Martin PMD, Hill GB. Cervical cancer in relation to tobacco and alcohol consumption in Lesotho, Southern Africa. Cancer Detect Prev 1984; 7: 109-14.

10 Kramer L. Faggots. New York: Simon and Shuster, 1978

11 Marmor M, Friedman-Kien A, Laubenstein L, et al. Risk factors for Kaposi's sarcoma in homosexual men. Lancet 1982; i: 1083-7.

12 Haverkos HW, Pinsky PF, Drotman P, Bregman DJ. Disease manifestation among homosexual men with AIDS: a possible role of nitrites in Kaposi's sarcoma. Sexually Transmitted Dis 1985; 12: 203-8.

13 Lauritsen J, Wilson H. Death rush: poppers and AIDS. New York: Pagan Press, 1986.

14 Watney S. Safer sex as community practice. In: Aggleton P, Davies P, Hart $\mathrm{G}$, eds. AIDS: Individual, cultural $\mathcal{E}$ policy dimensions. Basingstoke: Falmer, 1990: 19-33. 